\title{
Integer Binomial Plan: a Generalization on Factorials and Binomial Coefficients
}

\author{
Luís Dias Ferreira \\ Colégio Valsassina, Av. Teixeira da Mota \\ Quinta das Teresinhas, 1959-010 Lisboa, Portugal \\ Tel: 351-210-195-836 E-mail: luisdiasferreira@ clix.pt
}

\begin{abstract}
The primary goal of this paper is to achieve a simple generalization on binomial coefficients for all integer numbers and to introduce the concept of factorial $n$ of order $k$, which is presented in the sets of real numbers $n$ and integer $k$. Some applications of this concept are examined, featuring the consistency of its proposal, and a pair of theorems demonstrated. In generalising binomial coefficients to all integers, Pascal's Triangle is expanded to an Integer Binomial Plan, which displays remarkable properties.
\end{abstract}

Additionally, we stumble on the strange concept of semi-integer derivative of what we may call a $k$-derivable function.

Keywords: Factorials, Binomial numbers, Pascal's triangle, Gamma function

\section{Introduction}

Factorials and, mainly, binomial coefficients are usually defined only in a quite narrow frame: the set $\mathrm{N}_{0}$. This is certainly due to the 'concrete' applications of combinatorics, for instance to probability problems (Sage \& Melsa). As a consequence, we display binomial coefficients in a correspondent narrow slice, an octant of an whole plan (see section 5): the so-called Pascal's Triangle.

However the generalization of Newton binomial itself, by means of MacLaurin series, points out a 'natural' generalization of $\left(\begin{array}{l}n \\ k\end{array}\right)$ to every real number $n$; this is the path we'll use in section 3. But to do it - and, above all, to also generalize binomial coefficients to negative $k$ - we'll take advantage of the concept of 'incomplete factorials' or, generally speaking, factorials of order $k, n_{(k)} !$.

Of course, the gamma function $\Gamma(n+1)$ provides a generalization of factorials to real (or even complex) variables $n$. We'll use it in this paper. Why then isn't it enough? Because it doesn't apply to $n$ ! when $n$ is a negative integer; in these case (as we'll also conclude) $n !=\infty$. Now, in order to expand Pascal's Triangle, we need an operative definition which includes this case; I propose the concept and the handy notation of $n_{(k)} !$.

This concept appeared to me - and that's where it all began - connected to a seemingly 'childish' curiosity: the extention of the equality

$$
\left(\begin{array}{c}
n \\
k+1
\end{array}\right)=\frac{n-k}{k+1} \cdot\left(\begin{array}{l}
n \\
k
\end{array}\right)
$$

to $k+p$, in the frame of Pascal's Triangle. It immediately leaded to the concept and notation of $n_{(p)} !$, so that we're able to write, in a quite similar way:

$$
\left(\begin{array}{c}
n \\
k+p
\end{array}\right)=\frac{(n-k)_{(p)} !}{(k+p)_{(p)} !} \cdot\left(\begin{array}{l}
n \\
k
\end{array}\right)
$$

This is the Property 3 examined in section 5. I soon realized that $n_{(k)}$ ! given by the equation (1.a) may be easily applied to negative $n$; for instance, $(-5)_{(3)} !=(-5) \cdot(-6) \cdot(-7)=-210$. And this opened the door to subsequent generalizations, beginning with negative order number $k$ [equation (4)]. Apparently, it should be possible to coherently expand Pascal's Triangle to all integer $n$ and $k$, this is, to what I called the Integer Binomial Plan. But, to achieve this goal, the consistency of the idea needed to be rigorously examined. The result is the paper I present here. It may sound useless and appear solely as a mathematical elementary curiosity; but we never know where curiosities may lead us!...

\section{Factorial of order $k$}

Consider two integer numbers, $n$ and $k$, with $n \geq k \geq 0$, along with the usual definition of $n$ ! and the special case 0 ! = 1 (Wolfram 1). We'll define factorial $\boldsymbol{n}$ of order $\boldsymbol{k}$ by (read "factorial $n k$ ")

$$
n_{(k)} !=\frac{n !}{(n-k) !},
$$


or, for $n, k>0$,

$$
n_{(k)} !=\prod_{p=0}^{k-1}(n-p)=n \cdot(n-1) \cdot \ldots \cdot(n-k+1)
$$

in this case, $n_{(k)}$ ! is the product of the $k$ major values of $n$ !. In some problems we may have recourse to this 'incomplete factorial' instead of the product $n$ !. For instance, as it is well known, ${ }_{n} P_{k}=n_{(k)}$ ! is the number of ordered sets with $k$ elements which are subsets of a set with $n$ elements. In other issues, the symbol $n_{(k)}$ ! simplifies mathematical expressions and, above all, its concept permits important generalizations.

Remark the following cases, obtained from (1):

$$
\left\{\begin{array}{l}
n_{(0)} !=1 \\
n_{(n)} !=n ! \\
n_{(1)} !=n, \quad \text { for } n \neq 0
\end{array}\right.
$$

According to the definition given above, we may define the binomial coefficient $\left(\begin{array}{c}n \\ k\end{array}\right)$ by the expression

$$
\left(\begin{array}{c}
n \\
k
\end{array}\right)=\frac{n_{(k)} !}{k !}
$$

and so, applying it to the Newton binomial, we may write

$$
(1+x)^{n}=\sum_{k=0}^{n} \frac{n_{(k)} !}{k !} x^{k}
$$

We'll now extend the definition (1) to negative order numbers:

$$
n_{(-k)} !=\frac{n !}{(n+k) !}
$$

it will result, then,

$$
n_{(-k)} !=\left[\frac{(n+k) !}{n !}\right]^{-1}=\left[\frac{(n+k) !}{[(n+k)-k] !}\right]^{-1}
$$

or, according to (1),

$$
n_{(-k)} !=\frac{1}{(n+k)_{(k)} !}
$$

Remark that, since $n+k \geq k$ even for $k>n$, we'll consider this expression valid for all integer $k \geq 0$.

These are some examples:
a) $7_{(3)} !=\frac{7 !}{4 !}=7 \cdot 6 \cdot 5=210$;
b) $5_{(-2)} !=\frac{1}{7_{(2)} !}=\frac{1}{42}$;
c) $1_{(-k)} !=\frac{1}{(k+1)_{(k)} !}=\frac{1}{(k+1) !}$;
d) $0_{(-k)} !=\frac{1}{k !}$
e) $n_{(-1)} !=\frac{1}{n+1}$.

\section{Further generalization of factorial of order $k$ and of binomial coefficient}

The generalization of the Newton binomial to a real number $n$ is simply achieved by the development in MacLaurin series of the function $y=(1+x)^{n}$ (Piskounov, 1975):

$$
(1+x)^{n}=1+n x+\frac{n(n-1)}{1.2} x^{2}+\ldots+\frac{n(n-1) \ldots(n-k+1)}{k !} x^{k}+\ldots
$$


We may write, then,

$$
(1+x)^{n}=\frac{n_{(0)} !}{0 !} x^{0}+\frac{n_{(1)} !}{1 !} x^{1}+\frac{n_{(2)} !}{2 !} x^{2}+\ldots \frac{n_{(k)} !}{k !} x^{k}+\ldots,
$$

if we simply consider the equation (1.a) - and, by extension, (1) - valid for $n \in \mathrm{R}$ and $k \in \mathrm{N}_{0}$. Therefore, if we conformably generalize the definition (3) for binomial coefficient, we obtain for the binomial the general expression (Note 1)

$$
(1+x)^{n}=\sum_{k=0}^{+\infty}\left(\begin{array}{l}
n \\
k
\end{array}\right) \cdot x^{k}=\sum_{k=0}^{+\infty} \frac{n_{(k)} !}{k !} x^{k} .
$$

Remark that, if $n$ is a natural number, all terms of $n_{(k)}$ ! with $k>n$ result null because, for each one of them, the zero figures as one of the parcels (for instance, $\left.n_{(n+1)} !=n \cdot(n-1) \cdots \cdot 2 \cdot 0=0\right)$; in this case, we'll obtain the expression (3.a). Meanwhile, this reasoning leads to the conclusion that, $n$ and $k$ being natural numbers,

$$
k>n \Rightarrow n_{(k)} !=\frac{n !}{(n-k) !}=0
$$

but, since $n$ ! is also a natural number and $n-k<0$, the final conclusion is that, for any integer number $m$ :

$$
m<0 \Rightarrow \frac{1}{m !}=0 \text { or } m !=\infty .
$$

It's not difficult to understand this result if we conceive $(-n)$ ! as the product of $-n$ by the infinite integer numbers smaller than $-n$, none of them being equal to zero (Note 2). Remark that we deal here with a sign-indeterminate infinity because, as we go on multiplying $(-n) \cdot(-n-1) \cdots(-n-p) \cdot \ldots$, sign alternatively changes from positive to negative and vice-versa. Finally, this conclusion allows us to include the concept of binomial coefficient in the generalization above propounded to negative $k$, keeping the validity of expression (3) for all real $n$ and integer $k$. In this large sense, binomial coefficients must be seen as limits of a quotient.

As a matter of fact, generally speaking, factorials of order $k$-including complete factorials, $n !=n_{(n)} !-$ must also be seen as limits of a quotient. Furthermore, as presupposed in the generalization of $n_{(k)}$ !, if $k>0$ this factorial represents the product of the $k$ values presented in equation (1.a) even for $n<0$. For instance (Note 3):

$$
(-8)_{(3)} !=\frac{(-8) !}{(-11) !}=\frac{(-8) \cdot(-9) \cdot(-10) \cdot(-11) !}{(-11) !}=(-8) \cdot(-9) \cdot(-10)=-720 \text {; }
$$

if $k<0$, then, according to (4), $n_{(k)}$ ! represents the inverse of a product of $-k$ factors:

$$
(-8)_{(-3)} !=\frac{1}{(-5)_{(3)} !}=\frac{1}{(-5) \cdot(-6) \cdot(-7)}=-\frac{1}{210} .
$$

From now on, we'll be interested mainly in integer factorials and integer binomial coefficients (though many enouncements remain valid for a larger set of numbers). Remark that, for $n \neq 0$, we may write the well known identity [case b) of Theorem 1 right ahead]:

$$
n !=n \cdot(n-1) !
$$

Before proceeding, we must pay attention to a very important feature. Because of the limit nature of factorials and binomial coefficients, if $n_{(k)} !=0$ or $n_{(k)} !=\infty$ the equality transitive property isn't necessarily true; as a matter of fact, in general, it isn't and so, in these cases, we must be very careful. I mean, the fact that $n_{(k)}$ ! is a null or an infinite number doesn't mean that all numbers in one or the other of these circumstances are equal or even that the reason $n_{(k)} ! / n_{\left(k^{\prime}\right)}^{\prime} !$ is undetermined. On the contrary, this reason is a very precise number:

$$
\frac{n_{(k)} !}{n_{\left(k^{\prime}\right)}^{\prime} !}=\frac{n !}{n^{\prime} !} \cdot \frac{\left(n^{\prime}-k^{\prime}\right) !}{(n-k) !}
$$

For instance,

a) $(-6) !=\infty$ and $(-9) !=\infty$

but, nevertheless, $\frac{(-6) !}{(-9) !}=\frac{(-6) \cdot(-7) \cdot(-8) \cdot(-9) !}{(-9) !}=-8(3) !=-336 \quad$ or $\quad(-6) !=-336 \cdot(-9)$ ! .

b) $6_{(10)} !=\frac{6 !}{(-4) !}=0$ and $2(8) !=\frac{2 !}{(-6) !}=0$

but $\frac{6_{(10)} !}{2_{(8)} !}=\frac{6 !}{2 !} \cdot \frac{(-6) !}{(-4) !}=6_{(4)} ! \cdot \frac{(-6) !}{(-4) \cdot(-5) \cdot(-6) !}=\frac{6 \cdot 5 \cdot 4 \cdot 3}{(-4) \cdot(-5)}=18 \quad$ or $\quad 6_{(10)} !=18 \cdot 2_{(8)} !$. 
c) $0_{(5)} !=\frac{0 !}{(-5) !}=0$ and $4_{(5)} !=\frac{4 !}{(-1) !}=0$

but $\frac{0_{(5)} !}{4(5) !}=\frac{1}{4 !} \cdot \frac{(-1) !}{(-5) !}=1$ or $0_{(5)} !=4_{(5)} !$.

d) $5_{(8)} !=\frac{5 !}{(-3) !}=0$ and $2_{(8)} !=\frac{2 !}{(-6) !}=0$

but $\frac{5_{(8)} !}{2_{(8)} !}=\frac{5 !}{2 !} \cdot \frac{(-6) !}{(-3) !}=5_{(3)} ! \frac{(-6) !}{(-3) \cdot(-4) \cdot(-5) \cdot(-6) !}=-1 \quad$ or $\quad 5_{(8)} !=-2_{(8)} !$.

These last two cases establish an equality or an anti-equality relationship between two factorial of the same order. They obey to the theorem 2 enounced ahead, which generalizes the following remark: as we saw above, the number $(-8)_{(3)}$ ! may be written as

$$
(-8)_{(3)} !=(-8) \cdot(-9) \cdot(-10), \text { this is, } \quad(-8)_{(3)} !=-10_{(3)} !,
$$

in the same way that

$$
(-8)_{(4)} !=(-8) \cdot(-9) \cdot(-10) \cdot(-11)=11_{(4)} !
$$

or, in general, for $k>0,(-8)_{(k)} !=(-1)^{k} \cdot(8+k-1)_{(k)}$ !. This particular result, as well as its generalization (theorem 2 ), comes from the fact that, under certain circumstances, two factorials of the same order concern products with the same number of parcels which are equal in absolute values but sometimes result positive in one case and negative in the other.

Similar arguments may be presented for binomial coefficients (though these have always finite values). For instance, for

$$
\left\{\begin{array}{l}
a=\left(\begin{array}{l}
-5 \\
-2
\end{array}\right)=\frac{(-5) !}{(-3) !(-2) !}=\frac{(-5) \cdot(-4)}{(-2) !}=\frac{20}{(-2) !}=0 \text { and } \\
b=\left(\begin{array}{l}
6 \\
8
\end{array}\right)=\frac{6 !}{(-2) ! 8 !}=\frac{1}{42 \cdot(-2) !}=0
\end{array}\right.
$$

it results

$$
\frac{a}{b}=\frac{20}{(-2) !} \cdot 42 \cdot(-2) !=840
$$

To finalize this section I present two theorems concerning factorials of order $k$ for integer numbers. They have important applications, for instance in the next section (item 2), in binomial coefficients and in the Integer Binomial Plan (section 5).

Theorem 1. For integers $n, k$ and $p$, with some exceptions, we may write the following decomposition rule:

$$
n_{(k)} !=n_{(p)} !(n-p)_{(k-p)} !
$$

proof. As a matter of fact,

$$
n_{(k)} !=\frac{n !}{(n-k) !} \cdot \frac{(n-p) !}{(n-p) !}=n_{(p)} ! \frac{(n-p) !}{(n-k) !}
$$

this equality directly resulting in the equation (7). The mentioned exceptions concern in a way or another the zero, which stands isolated in the definition of factorial.

Remark the following particular cases:
a) $\quad p=1$ and $n \neq 0: \quad n_{(k)} !=n \cdot(n-1)_{(k-1)}$ ! (Note 4$)$
b) $\quad p=1$ and $k=n \neq 0: \quad n_{(n)} !=n \cdot(n-1)_{(n-1)}$ !
$\Rightarrow n !=n \cdot(n-1) !$
c) $p=n$ :
$n_{(k)} !=n ! 0_{(k-n)} !=n ! \frac{0 !}{(n-k) !}=\frac{n !}{(n-k) !}$
d) $p=k-1$ :
$n_{(k)} !=n_{(k-1)} !(n-k+1)$
e) $k=n$ :
$n !=n_{(p)} !(n-p) !$
and also
$(n+p) !=(n+p)_{(p)} ! n !$
f) $k=n+1$ :
$n_{(n+1)} !=n_{(p)} !(n-p)_{(n+1-p)} !=\frac{n !}{(-1) !}$
and so, for $n \geq 0$ : $\quad n_{(n+1)} !=0$. 
An example of this decomposition rule is

$$
9_{(5)} !=9_{(3)} ! \cdot 6_{(2)} !
$$

which is quite obvious. But the theorem also applies to negative order numbers; for instance:

$$
9_{(-5)} !=9_{(-3)} ! \cdot 12_{(-2)} !=\frac{1}{12_{(3)} !} \cdot \frac{1}{14_{(2)} !}=\frac{1}{12 \cdot 11 \cdot 10} \cdot \frac{1}{14 \cdot 13}=\frac{1}{14_{(5)} !},
$$

and this is correct, according to equation (4).

Theorem 2. For integer numbers $n$ and $k$, we have:

$$
n_{(k)} !=(-1)^{a} \cdot(k-n-1)_{(k)} !
$$

where

$$
a=k \quad \text { if }\left\{\begin{array} { l } 
{ n \geq 0 \quad \text { and } k \leq n } \\
{ n < 0 }
\end{array} \text { and } k > n ; \quad \text { or } a = k - 1 \quad \text { if } \left\{\begin{array}{l}
n \geq 0 \text { and } k>n \\
n<0
\end{array} \text { and } k \leq n .\right.\right.
$$

For a negative order number, according to (4), the equation (8) is equivalent to

$$
n_{(-k)} !=\frac{(-1)^{a}}{(-n-1)_{(k)} !} .
$$

proof. Note that the difference in the value of the exponent $a$ relates to the fact that $a=k$ concerns finite and non-null factorials $n_{(k)}$ !, whilst $a=k-1$ corresponds to the "extreme" possibilities (Note 5):

$$
\left\{\begin{array}{lll}
n \geq 0 \text { and } k>n & \Rightarrow & n_{(k)} !=\frac{n !}{(n-k) !}=0 \\
n<0 \text { and } k \leq n & \Rightarrow & n_{(k)} !=\frac{n !}{(n-k) !}=\infty
\end{array}\right.
$$

Let's see now, if patience is enough, a quite long proof of this theorem (Note 6), which is based on the concept of $n_{(k)}$ ! as the product (1.a) of $k$ factors, if $k>0$.

1. $n \geq 0$ and $k \leq n$ :

1.0) $k=0$ : we'll get, for whatever $n, n_{(0)} !=(-1)^{a} \cdot(-n-1)_{(0)} !$, this is $1=(-1)^{a} \cdot 1$ or $a=k=0$.

1.1) $k>0$ :

$$
\begin{aligned}
(k-n-1)_{(k)} ! & =\prod_{p=0}^{k-1}(k-n-1-p)= \\
& =(k-n-1) \cdot(k-n-2) \cdot \ldots \cdot(k-n-1-k+1)= \\
& =[-(n-k+1)] \cdot[-(n-k+2)] \cdot \ldots \cdot[-n] ;
\end{aligned}
$$

this last expression is formed exactly but in inverse order by the $k$ symmetrical elements of $n_{(k)}$ !; therefore, we may write $(n)_{(k)} !=(-1)^{k} \cdot(k-n-1)_{(k)} !$.

1.2) $k<0$ :

$$
n_{(k)} !=\frac{1}{(n-k)_{(-k)} !}, \quad \text { according to }(4)
$$

remark that $n-k \geq-k$ and so $(n-k)_{(-k)} ! \geq 1$ is a natural finite number. On the other hand, for the second term of the equation (8):

$$
(k-n-1)_{(k)} !=\frac{1}{(-n-1)_{(-k)} !} .
$$

Now, according to (1.a), the first denominator is

$$
(n-k)_{(-k)} !=(n-k) \cdot(n-k-1) \cdot \ldots \cdot(n+1),
$$

which is the product of $-k$ parcels, just like the second denominator:

$$
\begin{aligned}
(-n-1)_{(-k)} ! & =(-n-1) \cdot(-n-2) \cdots \cdots(-n+k)= \\
& =[-(n+1)] \cdot[-(n+2)] \cdot \ldots \cdot[-n-k]= \\
& =(-1)^{k} \cdot(n+1) \cdot(n+2) \cdot \ldots \cdot(n-k)= \\
& =(-1)^{k} \cdot(n-k)_{(-k)} !,
\end{aligned}
$$

and this means, once again, that $(n)_{(k)} !=(-1)^{k} .(k-n-1)_{(k)} !$. 
2. $n<0$ and $k>n$ :

2.0) $k=0$ : like in 1.0), both factorials in (8) are equal to 1 , and also $a=k=0$.

2.1) $k>0$ : the reasoning and the conclusion are exactly the same than in 1.1).

2.2) $k<0$ :

$$
n_{(k)} !=\frac{1}{(n-k)_{(-k)} !} \quad \text { and } \quad(k-n-1)_{(k)} !=\frac{1}{(-n-1)_{(-k)} !} .
$$

Now, $(n-k)<0$ and $(-n-1) \geq 0$; since $-k>0$, there is at least one parcel in the denominators factorials. Consequently, we are conduced to the same conclusion than in 1.2).

3. $n \geq 0$ and $k>n$ :

$$
\text { By definition, } \quad n_{(k)} !=\frac{n !}{(n-k) !} \quad \text { and } \quad(k-n-1)_{(k)} !=\frac{(k-n-1) !}{(-n-1) !} .
$$

Now, by hypothesis, $n \geq 0$ and $(k-n-1) \geq 0$; on the other hand, $(n-k)<0$ and $(-n-1)<0$. So, both factorials are null; but we may write

$$
\frac{n_{(k)} !}{(k-n-1)_{(k)} !}=\frac{N}{D},
$$

where

$$
N=\frac{n !}{(k-n-1) !} \quad \text { is the numerator (the quotient of non null finite factorials); }
$$

and

$$
D=\frac{(n-k) !}{(-n-1) !} \quad \text { is the denominator (the quotient of infinite factorials). }
$$

Let us express $N$ as a factorial of order $p$ :

$$
N=n_{(p)} !=\frac{n !}{(k-n-1) !} \quad \Rightarrow \quad p=2 n-k+1 ;
$$

in the same way, we may express $D$ as a factorial of order $q$ :

$$
D=(n-k)_{(q)} !=\frac{(n-k) !}{(-n-1) !} \quad \Rightarrow \quad q=2 n-k+1=p .
$$

$\frac{N}{D}$ expresses, therefore, the reason between factorials of the same order $p$ :

$$
\frac{n_{(k)} !}{(k-n-1)_{(k)} !}=\frac{n_{(p)} !}{(n-k)_{(p) !}}, \quad \text { considering } p=2 n-k+1 .
$$

Remark that, for $n=0$, it comes

$$
\frac{N}{D}=\frac{1}{(k-1) !} \cdot \frac{(-1) !}{(-k) !}=\frac{1}{(k-1) !} \cdot \frac{(-1) \cdot(-2) \cdot \ldots \cdot(-k+1) \cdot(-k) !}{(-k) !}=\frac{(-1)^{k-1} \cdot(k-1) !}{(k-1) !}=(-1)^{k-1} \Rightarrow a=k-1 .
$$

We'll consider now $n>0$.

3.a If $p=0$, this is, $k=2 n+1$ (an odd number), we are conduced to a tautology because, in this case, $k-n-1=n$ and so $n_{(k)} !=(-1)^{a} \cdot n_{(k)}$ ! obliges the exponent $a$ to be even, which certainly occurs for $a=k-1=2 n$.

3.b If $p>0$ (this is, $k<2 n+1$ ), both $N$ and $D$ consist on the product of $p$ factors. But

$$
\begin{aligned}
n_{(p)} ! & =n \cdot(n-1) \cdot \ldots \cdot[n-(2 n-k+1)+1]= \\
& =n \cdot(n-1) \cdot \ldots \cdot(k-n)
\end{aligned}
$$

(with $k-n>0$ ) and

$$
\begin{aligned}
(n-k)_{(p)} ! & =(n-k) \cdot(n-k-1) \cdot \ldots \cdot[n-k-(2 n-k+1)+1]= \\
& =[-(k-n)] \cdot[-(k-n+1)] \cdot \ldots \cdot[-n] ;
\end{aligned}
$$

remark that this is the product, in inverse order, of the symmetrical $p$ factors of $n_{(p)}$ !; and this means that

$$
\frac{n_{(k)} !}{(k-n-1)_{(k)} !}=(-1)^{2 n-k+1}=(-1)^{k-1} \Rightarrow a=k-1 \text {. }
$$


3.c If $p<0$, then $n_{(p)}$ ! and $(n-k)_{(p)}$ ! may be expressed, according to (4), as

$$
n_{(p)} !=\frac{1}{(n-p)_{(-p)} !} \quad \text { and } \quad(n-k)_{(p)} !=\frac{1}{(n-k-p)_{(-p)} !}
$$

resulting

$$
\frac{n_{(k)} !}{(k-n-1)_{(k)} !}=\frac{(n-k-p)_{(-p)} !}{(n-p)_{(-p)} !}
$$

this is the quotient of two factorials of order $-p$ (positive). The reasoning, similar to the anterior item, conduces to a number $-p$ of symmetrical factors and again to the exponent $a=k-1$.

4. $n<0$ and $k \leq n$ :

Like before, we may write $\frac{n_{(k)} !}{(k-n-1)_{(k)} !}=\frac{N}{D}$, considering now

$$
N=\frac{(-n-1) !}{(n-k) !} \quad \text { (the quotient of non null finite factorials); }
$$

and

$$
D=\frac{(k-n-1) !}{n !} \quad \text { (the quotient of infinite factorials). }
$$

We may also express $N$ and $D$ as factorials of the same order $p$ :

$$
\begin{array}{ccc}
N=(-n-1)_{(p)} ! & \Rightarrow & p=-2 n+k-1 ; \\
D=(k-n-1)_{(q)} ! & \Rightarrow & q=-2 n+k-1=p .
\end{array}
$$

If we suppose $p>0, N$ and $D$ express the product of $p$ factors inversely symmetrical:

$$
\begin{aligned}
N & =(-n-1) \cdot(n-2) \cdot \ldots \cdot[-n-k-1(-2 n+k-1)+1]= \\
& =[-(n+1)] \cdot[-(n+2)] \cdot \ldots \cdot[-(k-n-1)] ; \\
D & =(k-n-1) \cdot(k-n-2) \cdot \ldots \cdot(n+1)
\end{aligned}
$$

Remark that it must be $n+1>0$; as a matter of fact, if $n+1 \leq 0$, then $p=k+1 \leq 0$, and this concerns the two following hypothesis. So,

$$
\frac{N}{D}=\frac{n_{(k)} !}{(k-n-1)_{(k)} !}=(-1)^{-2 n+k-1}=(-1)^{k-1} . \Rightarrow a=k-1 .
$$

Alike before, we obtain a tautology and $a=k-1$ for $p=0$; and, once again, if $p<0$, the problem may be resolved applying (4) to $N$ and to $D$, finally resulting $\frac{N}{D}=(-1)^{k-1}$.

\section{Some applications}

We'll take a look now at some different problems concerning direct applications - and natural extensions - of the concepts presented in the precedent sections. This allows us to test their consistency and pertinence.

1. If $y=x^{n}$, we obtain for the derivative of order $k$ of $y$ relatively to $x$ the generic expression

$$
y^{(k)}=n_{(k)} ! x^{n-k}
$$

which relates the derivative with the factorial $n$ of order $k$. Remark that $y^{(0)}=n_{(0)} ! x^{n-0}=x^{n}=y$. We may suppose then that the integral $\int y d x$ should be resolved by the expression

$$
\int y d x=y^{(-1)}+C
$$

that is to say, according to (9) and example e) in section 1 ,

$$
\int y d x=n_{(-1)} ! x^{n+1}+C=\frac{x^{n+1}}{n+1}+C,
$$

this is, effectively, correct (for $n \neq-1$ ). In the same way, considering also $n \neq-2$,

$$
\int\left(\int y d x\right)=n_{(-2)} ! x^{n+2}+C^{\prime}=\frac{x^{n+2}}{(n+1)(n+2)}+C^{\prime} .
$$

On the other hand, for $n \in \mathrm{N}_{0}, y^{(n)}=n_{(n)} ! x^{0}=n$ !, which is a constant independent from $x$. As a consequence, all the derivatives of orders superior to $n$ must be null; this conclusion agrees with (1) and (6): $k>n \Rightarrow n_{(k)} !=0$. 
2. It comes from (9) that

$$
y=x^{n} \Rightarrow\left[y^{(k)}\right]^{(p)}=y^{(k+p)}=n_{(k+p)} ! x^{n-(k+p)} .
$$

As a matter of fact, if $w=y^{(k)}=n_{(k)} ! x^{n-k}$, then

$$
w^{(p)}=n_{(k)} !\left[x^{n-k}\right]^{(p)}=n_{(k)} !(n-k)_{(p)} ! x^{n-(k+p)} ;
$$

but $n_{(k)} !(n-k)_{(p)} !=n_{(k+p)} !$, according to the decomposition rule (7), and so we obtain the equation (9.a), presuming that each derivative is given by (9).

A particular case is the amusing identity

$$
\left[y^{(k)}\right]^{(-k)}=\left[y^{(-k)}\right]^{(k)}=y^{(0)}=y .
$$

3. Until now we have only considered integer order numbers; but the definition (1) also stands for non integer $k$. This extended definition, along with the gamma function and the equation (9), allows the introduction of the strange concept of positive or negative semi-integer derivative for $y=x^{n}$. For instance, making $k=1 / 2$, it results

$$
y=x^{n} \Rightarrow y^{\left(\frac{1}{2}\right)}=n_{\left(\frac{1}{2}\right)} ! x^{n-\frac{1}{2}}=\frac{n !}{\left(n-\frac{1}{2}\right) !} \sqrt{x^{2 n-1}} .
$$

Considering $n$ as a positive integer, we'll obtain the denominator factorial by applying the gamma function to semi-integer numbers (Wolfram 1):

$$
\left(n-\frac{1}{2}\right) !=\frac{\sqrt{\pi}}{2^{n}}(2 n-1) ! !,
$$

where $m$ !! is the double factorial of $m$ (Wolfram 2); for odd $m$ :

$$
m ! !=m \cdot(m-2) \ldots .5 \cdot 3 \cdot 1 ;
$$

we may write, then:

$$
y=x^{n} \Rightarrow y^{\left(\frac{1}{2}\right)}=\frac{2^{n}}{\sqrt{\pi}} \cdot \frac{n !}{(2 n-1) ! !} \sqrt{x^{2 n-1}} .
$$

In particular, if $n=1$, it comes from (10):

$$
y=x \Rightarrow y^{\left(\frac{1}{2}\right)}=\frac{1}{\left(\frac{1}{2}\right) !} \sqrt{x}=2 \sqrt{\frac{x}{\pi}},
$$

since (Wolfram 1) $\left(\frac{1}{2}\right) !=\frac{1}{2} \sqrt{\pi}$. For $n=2$, we'll obtain

$$
y^{\left(\frac{1}{2}\right)}=\frac{2 !}{\left(\frac{3}{2}\right) !} \sqrt{x^{3}}
$$

but $\left(\frac{3}{2}\right) !=\left(2-\frac{1}{2}\right) !=\frac{\sqrt{\pi}}{4} \cdot 3 ! !=\frac{3}{4} \sqrt{\pi}$ and so

$$
y=x^{2} \Rightarrow y^{\left(\frac{1}{2}\right)}=\frac{8}{3} \sqrt{\frac{x^{3}}{\pi}} .
$$

On the other hand, for the derivative of negative order $k=-1 / 2$ of $y=x^{n}$, it comes from (9) that $y^{\left(-\frac{1}{2}\right)}=\frac{n !}{\left(n+\frac{1}{2}\right) !} x^{\frac{2 n+1}{2}}$ or (Wolfram 1), since $\left(n+\frac{1}{2}\right) !=\frac{\sqrt{\pi}}{2^{n+1}}(2 n+1) ! !$ :

$$
y=x^{n} \Rightarrow y^{\left(-\frac{1}{2}\right)}=\frac{2^{n+1}}{\sqrt{\pi}} \cdot \frac{n !}{(2 n+1) ! !} \sqrt{x^{2 n+1}}
$$

therefore, in particular, making $n=1$ :

$$
y=x \Rightarrow y^{\left(-\frac{1}{2}\right)}=\frac{4}{3} \sqrt{\frac{x^{3}}{\pi}} .
$$


Frankly, I don't know what might be the sense or the use of semi-integer derivatives. Maybe it's just a fantasy. But consider the function $y=x$ and its semi-integer derivatives (10.b) and (11.a); we may see that, following (9), the equation (9.a) applies in these three particular cases:

$$
\begin{aligned}
& {\left[y^{\left(\frac{1}{2}\right)}\right]^{\left(\frac{1}{2}\right)}=\frac{2}{\sqrt{\pi}}\left[\left(\frac{1}{2}\right)_{\left(\frac{1}{2}\right)} ! x^{\frac{1}{2}-\frac{1}{2}}\right]=\frac{2}{\sqrt{\pi}}\left(\frac{1}{2}\right) !=\frac{2}{\sqrt{\pi}} \frac{\sqrt{\pi}}{2}=1=y^{(1)}} \\
& {\left[y^{\left(-\frac{1}{2}\right)}\right]^{\left(-\frac{1}{2}\right)}=\frac{4}{3 \sqrt{\pi}}\left[\left(\frac{3}{2}\right)_{\left(-\frac{1}{2}\right)} ! x^{\frac{3}{2}+\frac{1}{2}}\right]=\frac{4}{3 \sqrt{\pi}}\left[\left(\frac{3}{2}\right) ! \frac{x^{2}}{2}\right]=\frac{4}{3 \sqrt{\pi}}\left[\frac{3 \sqrt{\pi}}{4} \frac{x^{2}}{2}\right]=\frac{x^{2}}{2}=y^{(-1)} ; \quad \text { and }} \\
& {\left[y^{\left(\frac{1}{2}\right)}\right]^{\left(-\frac{1}{2}\right)}=\frac{2}{\sqrt{\pi}}\left[\left(\frac{1}{2}\right)_{\left(-\frac{1}{2}\right)} ! x^{\frac{1}{2}+\frac{1}{2}}\right]=\frac{2}{\sqrt{\pi}}\left(\frac{1}{2}\right) ! x=\frac{2}{\sqrt{\pi}} \frac{\sqrt{\pi}}{2} x=x=y^{(0)} \quad \text { or } \quad\left[y^{\left(-\frac{1}{2}\right)}\right]^{\left(\frac{1}{2}\right)}=y^{(0)} .}
\end{aligned}
$$

Finally, remark that, if we consider $a=x^{2}$ and $b=x$, then, for an integer $k$ :

$$
\left\{\begin{array}{l}
a^{(k)}=2_{(k)} ! x^{2-k} \\
b^{(k-1)}=1_{(k-1)} ! x^{2-k} ;
\end{array} \quad \text { but } 2_{(k)} !=\frac{2}{(2-k) !} \text { and } 1_{(k-1)} !=\frac{1}{(2-k) !}, \quad \text { which means that } a^{(k)}=2 b^{(k-1)} ;\right.
$$

coherently, this equality also stands for $k=1 / 2$ :

$$
a^{\left(\frac{1}{2}\right)}=\frac{8}{3} \sqrt{\frac{x^{3}}{\pi}}=2 \cdot \frac{4}{3} \sqrt{\frac{x^{3}}{\pi}}=2 b^{\left(-\frac{1}{2}\right)} .
$$

NOTE: Like for $y=x^{n}$, there are other functions $y=f(x)$ for which we may express their derivative of order $k$ as a function of $k$; we'll call them $k$-derivable functions. In this case, we may often obtain the correspondent integral as a derivative of order -1 (plus an arbitrary constant). We may, as well, express semi-integer derivatives in an equation.

Two examples:

$$
\begin{aligned}
& y=\sin x \quad \Rightarrow \quad y^{(k)}=\sin \left(x+k \frac{\pi}{2}\right) ; \quad \int \sin x=y^{(-1)}+C=\cos x+C ; \quad y^{\left(\mp \frac{1}{2}\right)}=\sin \left(x \pm \frac{\pi}{4}\right) ; \\
& y=e^{x} \quad \Rightarrow \quad y^{(k)}=y^{(0)}=e^{x} .
\end{aligned}
$$

4. Applying the development in MacLaurin series, expression (5), we obtain

$$
\frac{1}{1+x}=(1+x)^{-1}=\sum_{k=0}^{n}\left(\begin{array}{c}
-1 \\
k
\end{array}\right) x^{k}=\sum_{k=0}^{+\infty} \frac{(-1)_{(k)} !}{k !} x^{k}
$$

now, according to (8) (considering for the exponent $a=k$ since $n<1$ and $k>n$ ), it must be

$$
(-1)_{(k)} !=(-1)^{k} \cdot(1+k-1)_{(k)} !=(-1)^{k} \cdot k !
$$

and therefore

$$
\frac{1}{1+x}=\sum_{k=0}^{+\infty}(-x)^{k}
$$

One must notice that this series is convergent only for $|x|<1$; it results, then,

$$
\frac{1}{1+x}=1-x+x^{2}-x^{3}+\ldots+(-x)^{k}+\ldots
$$

5. Applying once again the development in MacLaurin series for the function $y=(1+x)^{n}$, considering now $x=1$, we obtain

$$
\sum_{k=0}^{+\infty}\left(\begin{array}{l}
n \\
k
\end{array}\right)=2^{n}
$$

Remark that this result is valid only when the series is convergent, which doesn't always happen.

For instance, making $n=1 / 2$ in (12), we obtain the following equality

$$
\sqrt{2}=\sum_{k=0}^{+\infty}\left(\begin{array}{c}
1 / 2 \\
k
\end{array}\right)=\sum_{k=0}^{+\infty} \frac{(1 / 2)_{k} !}{k !}=1+\frac{1}{2}-\frac{1}{8}+\frac{1}{16}-\frac{1}{128}+\ldots
$$

For a non negative integer $n$, all the second member terms in (12) for $k>n$ result null and the series transforms in the well known partial sum

$$
\sum_{k=0}^{n}\left(\begin{array}{l}
n \\
k
\end{array}\right)=2^{n}
$$


6. Consider the sequence of Fibonacci numbers, usually defined by $F_{n}=F_{(n-2)}+F_{(n-1)}$ (for $\left.n \geq 2\right)$ :

$$
\begin{array}{llllllllll}
1 & 1 & 2 & 3 & 5 & 8 & 13 & 21 & \ldots
\end{array}
$$

we may consider this as the right wing of Fibonacci numbers. As a matter of fact, we may use $F_{n}=F_{(n+2)}-F_{(n+1)}$ to obtain $F_{0}=0$ as a neutral element and all $F_{(-n)}=$ with $-n<0$ as the left wing of Fibonacci numbers. Alternatively we may use Binet's formula (Wolfram 3)

$$
F_{n}=\frac{(1+\sqrt{5})^{n}-(1-\sqrt{5})^{n}}{2^{n} \sqrt{5}}
$$

which relates the Fibonnaci numbers to the golden ratio. Remark that

$$
F_{(-n)}=\frac{2^{n}}{\sqrt{5}}\left[\frac{1}{(1+\sqrt{5})^{n}}-\frac{1}{(1-\sqrt{5})^{n}}\right]=-(-1)^{n} \frac{(1+\sqrt{5})^{n}-(1-\sqrt{5})^{n}}{2^{n} \sqrt{5}} \Rightarrow F_{(-n)}=(-1)^{n+1} F_{n} .
$$

As a conclusion, we may consider the complete sequence as having the form

$$
\begin{array}{lllllllllllllllllllll}
\ldots & -21 & 13 & -8 & 5 & -3 & 2 & -1 & 1 & 0 & 1 & 1 & 2 & 3 & 5 & 8 & 13 & 21 & \ldots
\end{array}
$$

The point here is that, using MacLaurin series, we may write Binet's formula as a function of binomial coefficients; in fact,

$$
\left\{\begin{array}{l}
(1+\sqrt{5})^{n}=\sum_{k=0}^{+\infty}\left(\begin{array}{l}
n \\
k
\end{array}\right)(\sqrt{5})^{k} \\
(1-\sqrt{5})^{n}=\sum_{k=0}^{+\infty}\left(\begin{array}{l}
n \\
k
\end{array}\right)(-1)^{k}(\sqrt{5})^{k}
\end{array}\right.
$$

Therefore,

$$
F_{n}=\frac{1}{2^{n} \sqrt{5}} \sum_{K=0}^{+\infty}\left(\begin{array}{l}
n \\
k
\end{array}\right)(\sqrt{5})^{k}\left[1-(-1)^{k}\right]
$$

but

$$
\begin{cases}1-(-1)^{k}=0 & \text { for all even } k \\ 1-(-1)^{k}=2 & \text { for all odd } k\end{cases}
$$

and this means that $F_{n}=\frac{1}{2^{n} \sqrt{5}} \sum_{i=0}^{+\infty} 2 \cdot\left(\begin{array}{c}n \\ 2 i+1\end{array}\right)(\sqrt{5})^{2 i+1}$, or

$$
F_{n}=\frac{1}{2^{n-1}} \sum_{i=0}^{+\infty} 5^{i}\left(\begin{array}{c}
n \\
2 i+1
\end{array}\right)
$$

Unfortunately, we cannot use this formula for $n<0$ because the series is divergent [but see equation (22) in section 5]. For $n \geq 0$, as we have seen, all $\left(\begin{array}{c}n \\ 2 i+1\end{array}\right)$ with $2 i+1>n$ result null. We may write, then:

$$
F_{n}=\frac{1}{2^{n-1}} \sum_{i=0}^{\text {int }\left(\frac{\mathrm{n}-1}{2}\right)} 5^{i}\left(\begin{array}{c}
n \\
2 i+1
\end{array}\right)
$$

where int $\left(\frac{\mathrm{n}-1}{2}\right)$ is the integer value of $\frac{n-1}{2}$. The 5 th element, for instance, is given by the sum of 3 factors:

$$
F_{5}=\frac{1}{2^{4}} \sum_{i=0}^{2} 5^{i}\left(\begin{array}{c}
5 \\
2 i+1
\end{array}\right)=\frac{1}{2^{4}}\left[5^{0}\left(\begin{array}{l}
5 \\
1
\end{array}\right)+5^{2}\left(\begin{array}{l}
5 \\
3
\end{array}\right)+5^{2}\left(\begin{array}{l}
5 \\
5
\end{array}\right)\right]=\frac{1}{16}(5+50+25)=\frac{80}{16}=5 .
$$

7. The equation $x !=20 \cdot(x-2)$ ! admits two solutions. As a matter of fact,

$$
\frac{x !}{(x-2) !}=20 \Rightarrow x_{(2)} !=20, \quad \text { this is, } \quad x(x-1)=20,
$$

which means that

$$
\left\{\begin{array}{l}
x=5, \text { or } \\
x=-4
\end{array}\right.
$$


In this kind of problem, one normally considers the first solution as the only one valid. However, the second one is also valid; the number 20 comes out as the limit of two infinite numbers quotient (Note 7):

$$
(-4)_{(-2)} !=\frac{(-4) !}{(-6) !}=(-4) \cdot(-5)=20 \text {. }
$$

8. Let us calculate the binomial coefficients $\left(\begin{array}{c}-5 \\ 4\end{array}\right)$ and $\left(\begin{array}{c}5 \\ -4\end{array}\right)$, which we may call symmetric ones. We'll have

$$
\left(\begin{array}{c}
-5 \\
4
\end{array}\right)=\frac{(-5)_{(4)} !}{4 !}=\frac{8_{(4)} !}{4 !}=\frac{8 \cdot 7 \cdot 6 \cdot 5}{4 \cdot 3 \cdot 2 \cdot 1}=70
$$

and

$$
\left(\begin{array}{c}
5 \\
-4
\end{array}\right)=\frac{5_{(-4)} !}{(-4) !}=\frac{1}{(-4) !} \cdot \frac{1}{9_{(4)} !}=0 \cdot \frac{1}{3024}=0 .
$$

This result is included in a generic conclusion, which admits some exceptions: if an integer binomial coefficient is null, its inverse is not, and vice-versa. This fact clearly displays in the Integer Binomial Plan (see next section): non-null octants are opposite to null ones and vice-versa.

\section{Integer Binomial Plan}

The application of integer $n$ and $k$ values to $\left(\begin{array}{l}n \\ k\end{array}\right)$ allows us to perform a coherent expansion of the well known Pascal's Triangle to a whole plan. We will call it Integer Binomial Plan (I.B.P.). In this plan, the value of each $\left(\begin{array}{c}n \\ k\end{array}\right)$ will be fixed at the 'point' determined by the 'coordinates' $n$ and $k$, the two axis of coordinates crossing at the origin $O=\left(\begin{array}{l}0 \\ 0\end{array}\right)$. It will be convenient to represent the $n n$ axis in the vertical, from top to bottom, and the $k k$ axis in the horizontal, from left to right (this means a rotation of $-90^{\circ}$ upon the usual Cartesian axis); with this convention, the Pascal's Triangle will keep its familiar aspect, in the inferior right octant.

$$
\begin{aligned}
& \ldots\left(\begin{array}{l}
-3 \\
-3
\end{array}\right) \quad\left(\begin{array}{l}
-3 \\
-2
\end{array}\right) \quad\left(\begin{array}{l}
-3 \\
-1
\end{array}\right) \quad\left(\begin{array}{c}
-3 \\
\mathbf{0}
\end{array}\right) \quad\left(\begin{array}{c}
-3 \\
1
\end{array}\right)\left(\begin{array}{c}
-3 \\
2
\end{array}\right)\left(\begin{array}{c}
-3 \\
3
\end{array}\right) \ldots \\
& \ldots\left(\begin{array}{l}
-2 \\
-3
\end{array}\right) \quad\left(\begin{array}{l}
-2 \\
-2
\end{array}\right) \quad\left(\begin{array}{l}
-2 \\
-1
\end{array}\right) \quad\left(\begin{array}{c}
-2 \\
\mathbf{0}
\end{array}\right)\left(\begin{array}{c}
-2 \\
1
\end{array}\right)\left(\begin{array}{c}
-2 \\
2
\end{array}\right)\left(\begin{array}{c}
-2 \\
3
\end{array}\right) \ldots \\
& \cdots\left(\begin{array}{l}
-1 \\
-3
\end{array}\right) \quad\left(\begin{array}{l}
-1 \\
-2
\end{array}\right) \quad\left(\begin{array}{l}
-1 \\
-1
\end{array}\right) \quad\left(\begin{array}{c}
-1 \\
\mathbf{0}
\end{array}\right)\left(\begin{array}{c}
-1 \\
1
\end{array}\right)\left(\begin{array}{c}
-1 \\
2
\end{array}\right)\left(\begin{array}{c}
-1 \\
3
\end{array}\right) \ldots \\
& \cdots\left(\begin{array}{c}
\mathbf{0} \\
-\mathbf{3}
\end{array}\right) \quad\left(\begin{array}{c}
\mathbf{0} \\
-\mathbf{2}
\end{array}\right) \quad\left(\begin{array}{c}
\mathbf{0} \\
-\mathbf{1}
\end{array}\right) \quad\left(\begin{array}{l}
\mathbf{0} \\
\mathbf{0}
\end{array}\right) \quad\left(\begin{array}{l}
\mathbf{0} \\
\mathbf{1}
\end{array}\right) \quad\left(\begin{array}{l}
\mathbf{0} \\
\mathbf{2}
\end{array}\right) \quad\left(\begin{array}{l}
\mathbf{0} \\
\mathbf{3}
\end{array}\right) \ldots \\
& \cdots\left(\begin{array}{c}
1 \\
-3
\end{array}\right) \quad\left(\begin{array}{c}
1 \\
-2
\end{array}\right) \quad\left(\begin{array}{c}
1 \\
-1
\end{array}\right) \quad\left(\begin{array}{l}
\mathbf{1} \\
\mathbf{0}
\end{array}\right) \quad\left(\begin{array}{l}
1 \\
1
\end{array}\right) \quad\left(\begin{array}{l}
1 \\
2
\end{array}\right) \quad\left(\begin{array}{l}
1 \\
3
\end{array}\right) \ldots \\
& \cdots\left(\begin{array}{c}
2 \\
-3
\end{array}\right) \quad\left(\begin{array}{c}
2 \\
-2
\end{array}\right) \quad\left(\begin{array}{c}
2 \\
-1
\end{array}\right) \quad\left(\begin{array}{l}
2 \\
\mathbf{0}
\end{array}\right) \quad\left(\begin{array}{l}
2 \\
1
\end{array}\right) \quad\left(\begin{array}{l}
2 \\
2
\end{array}\right) \quad\left(\begin{array}{l}
2 \\
3
\end{array}\right) \ldots \\
& \ldots\left(\begin{array}{c}
3 \\
-3
\end{array}\right) \quad\left(\begin{array}{c}
3 \\
-2
\end{array}\right) \quad\left(\begin{array}{c}
3 \\
-1
\end{array}\right) \quad\left(\begin{array}{l}
3 \\
\mathbf{0}
\end{array}\right) \quad\left(\begin{array}{l}
3 \\
1
\end{array}\right) \quad\left(\begin{array}{l}
3 \\
2
\end{array}\right) \quad\left(\begin{array}{l}
3 \\
3
\end{array}\right) \ldots
\end{aligned}
$$

Concerning the values on the axis, we can easily conclude that:

- axis $O n:\left(\begin{array}{l}n \\ 0\end{array}\right)=1$;

- axis $O k:\left(\begin{array}{l}0 \\ k\end{array}\right)=0$, with the exception of $\left(\begin{array}{l}0 \\ 0\end{array}\right)=1$. 
On its turn, concerning the main diagonals, we have (considering $a>0$ ):

- 1st diagonal: $\left(\begin{array}{l}a \\ a\end{array}\right)=1$;

- 2nd diagonal: $\left(\begin{array}{c}-a \\ a\end{array}\right)=\frac{(-a)_{(a)} !}{a !}=\frac{(-1)^{a} \cdot(2 a-1)_{(a)} !}{a !}$;

- 3 rd diagonal: $\left(\begin{array}{c}-a \\ -a\end{array}\right)=1$

- 4th diagonal: $\left(\begin{array}{c}a \\ -a\end{array}\right)=0$.

Placing each numerical value of $\left(\begin{array}{l}n \\ k\end{array}\right)$ in its respective place, the I.B.P. takes the aspect of the following table:

\begin{tabular}{|c|c|c|c|c|c|c|c|c|c|c|c|c|}
\hline$\ldots$ & $\ldots$ & $\ldots$ & $\ldots$ & $\ldots$ & $\cdots$ & 1 & $\ldots$ & $\cdots$ & $\ldots$ & $\ldots$ & $\ldots$ & $\ldots$ \\
\hline 1 & 0 & 0 & 0 & 0 & 0 & 1 & -6 & 21 & -56 & 126 & -252 & 462 \\
\hline-5 & 1 & 0 & 0 & 0 & 0 & 1 & -5 & 15 & -35 & 70 & -126 & 210 \\
\hline 10 & -4 & 1 & 0 & 0 & 0 & 1 & -4 & 10 & -20 & 35 & -56 & 84 \\
\hline-10 & 6 & -3 & 1 & 0 & 0 & 1 & -3 & 6 & -10 & 15 & -21 & 28 \\
\hline 5 & -4 & 3 & -2 & 1 & 0 & 1 & -2 & 3 & -4 & 5 & -6 & 7 \\
\hline-1 & 1 & -1 & 1 & -1 & 1 & 1 & -1 & 1 & -1 & 1 & -1 & 1 \\
\hline 0 & 0 & 0 & $\mathbf{0}$ & 0 & 0 & 1 & 0 & 0 & 0 & 0 & 0 & 0 \\
\hline 0 & 0 & 0 & 0 & 0 & $\mathbf{0}$ & 1 & 1 & 0 & 0 & 0 & 0 & 0 \\
\hline 0 & 0 & 0 & 0 & $\mathbf{0}$ & 0 & 1 & 2 & 1 & 0 & 0 & 0 & 0 \\
\hline 0 & 0 & 0 & $\mathbf{0}$ & 0 & 0 & 1 & 3 & 3 & 1 & 0 & 0 & 0 \\
\hline 0 & 0 & 0 & 0 & 0 & 0 & 1 & 4 & 6 & 4 & 1 & 0 & 0 \\
\hline 0 & 0 & 0 & 0 & 0 & 0 & 1 & 5 & 10 & 10 & 5 & 1 & 0 \\
\hline 0 & 0 & 0 & 0 & 0 & 0 & 1 & 6 & 15 & 20 & 15 & 6 & 1 \\
\hline
\end{tabular}

The I.B.P. axis and main diagonals divide it in four quadrants and eight octants, counted in the direct sense. The first octant is precisely the Pascal's Triangle, in the inferior part at the right of $n n$ axis.

Now, the following properties are valid in all the I.B.P., considering the respective exceptions.

Property 1. For all $n$ and $k$ :

$$
\left(\begin{array}{l}
n \\
k
\end{array}\right)=\left(\begin{array}{c}
n \\
n-k
\end{array}\right)
$$

proof. This equality immediately derives from the generalized definition of binomial coefficient.

In Pascal's Triangle, this property expresses a symmetry in each line; in the whole I.B.P., however, it reveals a more subtle and interesting connection, also a symmetry in lines but often with respect to axes and diagonals. For instance, all the 2 nd Quadrant is symmetrical (in relation to the vertical semi-axis) to the 6th Octant (in relation to the 3rd diagonal).

The next two equalities are examples of the current property:

$$
\left(\begin{array}{l}
-3 \\
-5
\end{array}\right)=\left(\begin{array}{c}
-3 \\
2
\end{array}\right)=6 \text { and }\left(\begin{array}{c}
5 \\
-2
\end{array}\right)=\left(\begin{array}{l}
5 \\
7
\end{array}\right)=0
$$


Property 2. We have

$$
\left(\begin{array}{l}
n \\
k
\end{array}\right)+\left(\begin{array}{c}
n \\
k+1
\end{array}\right)=\left(\begin{array}{l}
n+1 \\
k+1
\end{array}\right)
$$

except for $n=k=-1$; in this case, $\left(\begin{array}{l}n+1 \\ k+1\end{array}\right)=\left(\begin{array}{l}0 \\ 0\end{array}\right)=1$.

proof.

$$
\begin{aligned}
\left(\begin{array}{l}
n \\
k
\end{array}\right)+\left(\begin{array}{c}
n \\
k+1
\end{array}\right) & =\frac{n !}{k !(n-k) !}+\frac{n !}{(k+1) !(n-k-1) !}= \\
& =\frac{n !}{k !(n-k)(n-k-1) !}+\frac{n !}{(k+1) \cdot k !(n-k-1) !}=\frac{n ![(k+1)+(n-k)]}{(k+1) !(n-k) !}= \\
& =\frac{(n+1) \cdot n !}{(k+1) !(n-k) !}=\frac{(n+1) !}{(k+1) ![(n+1)-(k+1)] !}=\left(\begin{array}{c}
n+1 \\
k+1
\end{array}\right) .
\end{aligned}
$$

Remark that we use, in the demonstration, the equalities

$$
(n+1) \cdot n !=(n+1) ! \quad \text { and } \quad(k+1) \cdot k !=(k+1) !,
$$

which are not valid for $n=-1$ and $k=-1$ respectively. Therefore, the property doesn't apply to these values.

This property is the well known Pascal's formula, a recursive relation which allows us to "build" quite easily the Pascal's Triangle. It also permits to "build" the I.B.P. exactly in the same way. It's curious (but necessary, as we may conclude by studying the numerical values of the I.B.P.) that the property doesn't apply to $\left(\begin{array}{l}0 \\ 0\end{array}\right)$.

For instance:

$$
\left(\begin{array}{c}
-5 \\
4
\end{array}\right)+\left(\begin{array}{c}
-5 \\
5
\end{array}\right)=\left(\begin{array}{c}
-4 \\
5
\end{array}\right) \text { or } 70+(-126)=-56
$$

Remark that (14) implies that, except for $n=k=-1$,

$$
\sum_{k=-\infty}^{+\infty}\left(\begin{array}{c}
n+1 \\
k+1
\end{array}\right)=\sum_{k=-\infty}^{+\infty}\left(\begin{array}{l}
n \\
k
\end{array}\right)+\sum_{k=-\infty}^{+\infty}\left(\begin{array}{c}
n \\
k+1
\end{array}\right) \Rightarrow \sum_{k=-\infty}^{+\infty}\left(\begin{array}{c}
n+1 \\
k
\end{array}\right)=2 \sum_{k=-\infty}^{+\infty}\left(\begin{array}{c}
n \\
k
\end{array}\right) .
$$

For $n<0$, this equation compares two infinities. But not for $n \geq 0$; in this case, the expression (12.a) is related to (16) because we obtain (Note 8 )

$$
\sum_{k=-\infty}^{+\infty}\left(\begin{array}{c}
n+1 \\
k
\end{array}\right)=\sum_{k=0}^{n+1}\left(\begin{array}{c}
n+1 \\
k
\end{array}\right)=2 \sum_{k=0}^{n}\left(\begin{array}{l}
n \\
k
\end{array}\right)=2 \cdot 2^{n}=2^{n+1}
$$

in fact, we should obtain (12.a) by recursevely appliyng these result starting from $n=0$.

\section{Property 3.}

$$
\left(\begin{array}{c}
n \\
k+p
\end{array}\right)=\frac{(n-k)_{(p)} !}{(k+p)_{(p)} !} \cdot\left(\begin{array}{l}
n \\
k
\end{array}\right)
$$

proof.

$$
\begin{aligned}
\frac{(n-k)_{(p)} !}{(k+p)_{(p)} !} \cdot\left(\begin{array}{l}
n \\
k
\end{array}\right) & =\frac{(n-k) !}{(n-k-p) !} / \frac{(k+p) !}{k !} \cdot \frac{n !}{k !(n-k) !}= \\
& =\frac{n !}{(k+p) !(n-k-p) !}=\left(\begin{array}{c}
n \\
k+p
\end{array}\right) .
\end{aligned}
$$

In fact, this property relates to the decomposition rule (7) enounced by the theorem 1 . If we make $p=1$ in equation (16), we'll obtain a well known equality in Pascal's Triangle (and, of course in the I.B.P.) (Note 9):

$$
\left(\begin{array}{c}
n \\
k+1
\end{array}\right)=\frac{n-k}{k+1} \cdot\left(\begin{array}{l}
n \\
k
\end{array}\right)
$$

It would seem quite evident to assume that (16) wouldn't apply if $\left(\begin{array}{l}n \\ k\end{array}\right)=0$ but, in fact, it does! There are no exceptions for it. In fact, the property (16), "innocuous" in Pascal's Triangle, brings forward a mathematical fact at the first sight bizarre, 
resulting from the already mentioned limit nature of factorials: the violation of an algebraic basic rule for multiplication (Note 10); this is, the product of a number $q$ by a null binomial coefficient isn't necessarily equal to zero:

$$
\left(\begin{array}{l}
n \\
k
\end{array}\right)=0 \Rightarrow q \cdot\left(\begin{array}{l}
n \\
k
\end{array}\right)\left\{\begin{array}{l}
=0 \\
\neq 0
\end{array}\right.
$$

For instance,

$$
\left(\begin{array}{l}
-5 \\
-2
\end{array}\right)=0, \quad \text { but } \quad\left(\begin{array}{c}
-5 \\
5
\end{array}\right)=\frac{(-3)_{(7)} !}{55_{(7)} !} \cdot\left(\begin{array}{c}
-5 \\
-2
\end{array}\right)=\frac{\frac{(-3) !}{(-10) !}}{\frac{5 !}{(-2) !}} \cdot \frac{(-5) !}{(-2) !(-3) !}=\frac{(-5)_{(5)} !}{5 !}=-126
$$

On the other hand,

$$
\left(\begin{array}{c}
5 \\
-6
\end{array}\right)=0, \quad \text { and } \quad\left(\begin{array}{c}
5 \\
-4
\end{array}\right)=\frac{11_{(2)} !}{(-4)_{(2)} !} \cdot\left(\begin{array}{c}
5 \\
-6
\end{array}\right)=\frac{\frac{11 !}{9 !}}{\frac{(-4) !}{(-6) !}} \cdot \frac{5 !}{(-6) ! \cdot 11 !}=\frac{5 !}{9 !} \cdot \frac{1}{(-4)} !=0
$$

\section{Property 4.}

$$
\left(\begin{array}{c}
n+p \\
k+p
\end{array}\right)=\frac{(n+p)_{(p)} !}{(k+p)_{(p)} !}\left(\begin{array}{l}
n \\
k
\end{array}\right)
$$

proof. This property relates binomials coefficients belonging to a same descendent diagonal. It is a direct application of the particular case e) of theorem 1:

$$
\left(\begin{array}{l}
n+p \\
k+p
\end{array}\right)=\frac{1}{(n-k) !} \frac{(n+p) !}{(k+p) !}=\frac{(n+p)_{(p)} ! n !}{(k+p)_{(p)} ! k !} \frac{1}{(n-k) !}=\frac{(n+p)_{(p)} !}{(k+p)_{(p)} !}\left(\begin{array}{l}
n \\
k
\end{array}\right) .
$$

Property 5.

$$
\left(\begin{array}{l}
n \\
k
\end{array}\right)=(-1)^{a} \cdot\left(\begin{array}{c}
k-n-1 \\
k
\end{array}\right)
$$

where

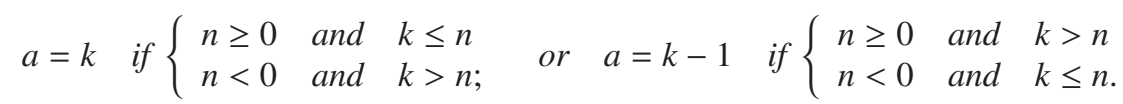

proof. The demonstration is simple and direct, following Theorem 2:

$$
\left(\begin{array}{l}
n \\
k
\end{array}\right)=\frac{n_{(k)} !}{k !}=\frac{(-1)^{a} \cdot(k-n-1)_{(k)} !}{k !}=(-1)^{a} \cdot\left(\begin{array}{c}
k-n-1 \\
k
\end{array}\right) .
$$

For instance:
a) $\left(\begin{array}{l}5 \\ 3\end{array}\right)=(-1)^{3} \cdot\left(\begin{array}{c}-3 \\ 3\end{array}\right)=(-1) \cdot(-10)=10 \quad($ exponent $a=k)$;
b) $\left(\begin{array}{l}-4 \\ -4\end{array}\right)=(-1)^{-5} \cdot\left(\begin{array}{l}-1 \\ -4\end{array}\right)=(-1) \cdot(-1)=1 \quad$ (exponent $\left.a=k-1\right)$;
c) $\left(\begin{array}{c}-6 \\ -11\end{array}\right)=(-1)^{-12} \cdot\left(\begin{array}{c}-6 \\ -11\end{array}\right)=1 \cdot(-252)=-252, \quad$ this being a tautology.

The equation (19) lays down perhaps the most remarkable feature of the I.B.P., which is enounced in two points:

1. If we use a fixed value for $n$ and make $k$ vary, the Property 5 transforms lines into diagonals (and vice-versa, of course).

The respective line and diagonal intersect at the point $\left(\begin{array}{l}n \\ k\end{array}\right)$ for

$$
k=2 n+1
$$

$k$ is, therefore, at that point, always an odd number. Remark that

$$
\left\{\begin{array}{l}
n \geq 0 \quad \Rightarrow \quad k>n \\
n<0 \Rightarrow k \leq n
\end{array}\right.
$$


so that, in every possible case, the exponent one must consider in (17) is $a=k-1$, thus resulting the tautology

$$
\left(\begin{array}{l}
n \\
k
\end{array}\right)=(-1)^{2 n} \cdot\left(\begin{array}{l}
n \\
k
\end{array}\right)
$$

As an example, in the following table, are thrown in relief the lines and diagonals obtained for a varying $k$ to

$$
n=-4 \text { and } n=5 \text {; }
$$

The line/diagonal intersection points are respectively:

$$
\left(\begin{array}{l}
-4 \\
-7
\end{array}\right)=-20 \text { and }\left(\begin{array}{c}
5 \\
11
\end{array}\right)=0
$$

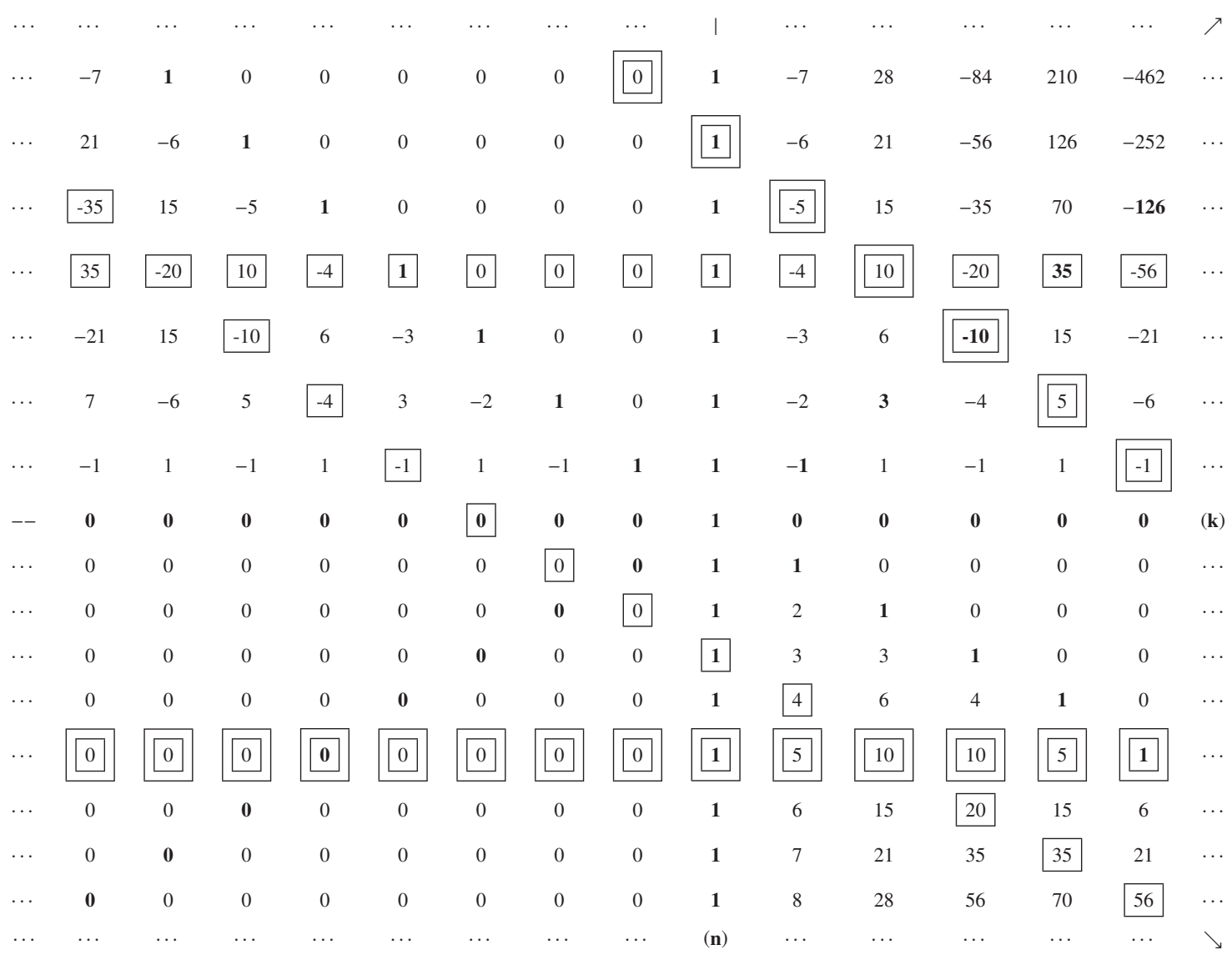

Consider the intersection point of corresponding line and diagonal for a given $n_{0}$ :

$$
\left(\begin{array}{l}
n_{0} \\
k_{0}
\end{array}\right) \quad \text { with } \quad k_{0}=2 n_{0}+1
$$

we may call it anchor point of these line and diagonal. Remark that applying successively the first and the fifth properties, we obtain

$$
\left(\begin{array}{l}
n_{0} \\
k_{0}
\end{array}\right)=\left(\begin{array}{c}
n_{0} \\
-n_{0}-1
\end{array}\right)=(-1)^{-n_{0}-1}\left(\begin{array}{c}
-2 n_{0}-2 \\
-n_{0}-1
\end{array}\right)=(-1)^{-n_{0}-1}\left(\begin{array}{c}
n_{0}^{\prime} \\
k_{0}^{\prime}
\end{array}\right)
$$

This $\left(\begin{array}{c}n_{0}^{\prime} \\ k_{0}^{\prime}\end{array}\right)$ is a point of the same descending diagonal than $\left(\begin{array}{l}n_{0} \\ k_{0}\end{array}\right)$ at a 'distance' $p_{0}$ :

$$
\left\{\begin{array}{l}
n_{0}^{\prime}=n_{0}+p_{0} \\
k_{0}^{\prime}=k_{0}+p_{0}
\end{array} \quad \text { where } \quad p_{0}=-3 n_{0}-2\right.
$$


We'll say that it is the 'image' of the 'anchor point' at the diagonal. Finally, you may observe in the I.B.P. displayed above that (except for $n_{0}=-1$ ) there is a relationship of equality or anti-equality between non-null binomial coefficients (mutual 'image points') of this diagonal equidistant one from the anchor-point and the other from this one's 'image'; I suppose that the exponent for $(-1)^{a}$ is the same $-n_{0}-1$ and so

$$
\left(\begin{array}{l}
n_{0}+p \\
k_{0}+p
\end{array}\right)=(-1)^{-n_{0}-1}\left(\begin{array}{c}
n_{0}^{\prime}-p \\
k_{0}^{\prime}-p
\end{array}\right) .
$$

2. If, on the other hand, we consider $k$ as a fixed value and make $n$ vary, the Property 4 sets a correspondence, two by two, between elements of the same column, as exemplified below for

$$
k=-8 ; \quad k=-5 ; \quad k=1 ; \quad k=4
$$

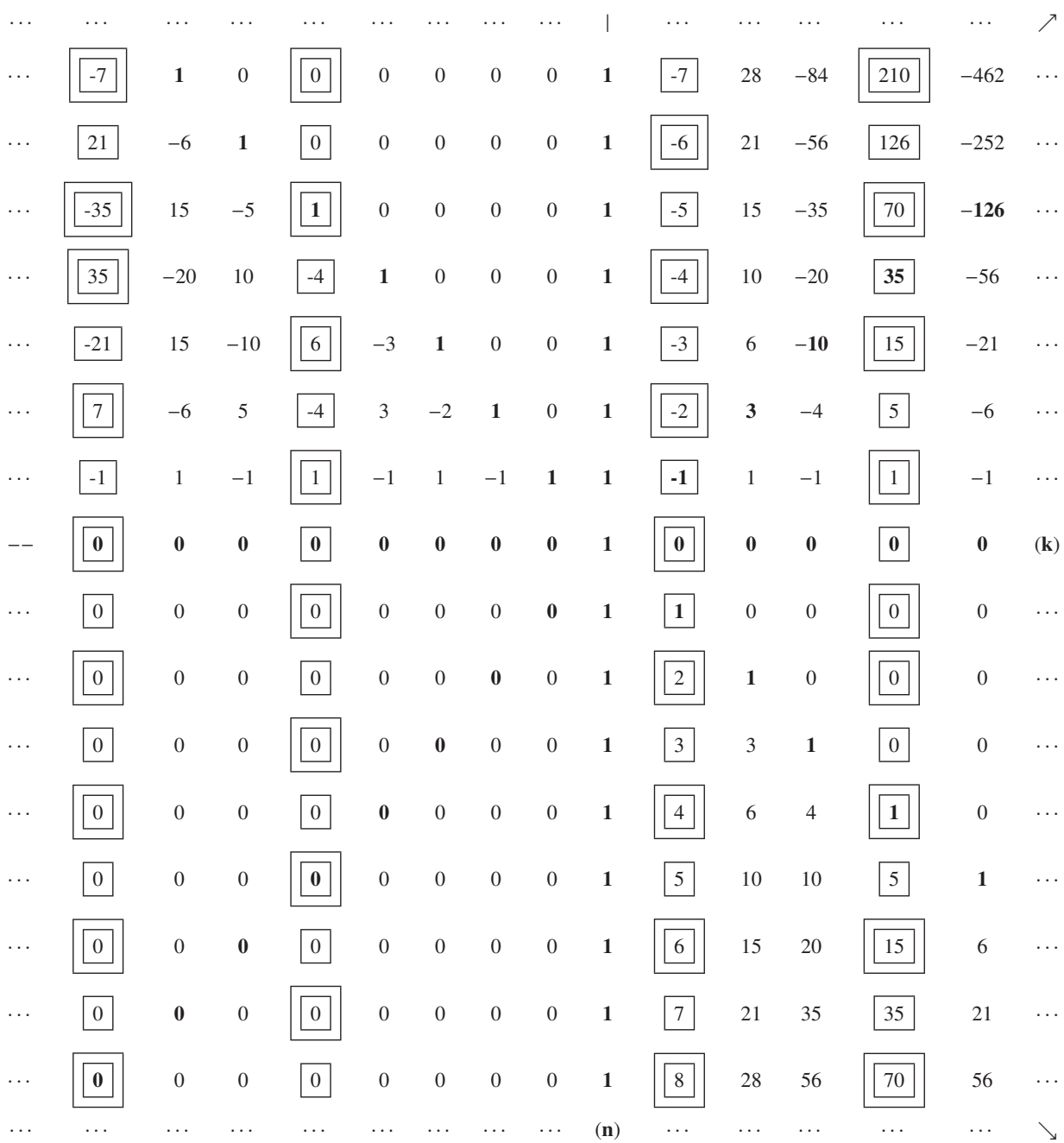

Other Properties. There are several other properties (in general, related to these first ones) in this amazing 'infinite square'. To finish this paper, I just want to present two or three of them, which I will not demonstrate.

a) We saw in item 6 of the precedent section an equation that relates the numbers of Fibonacci sequence with lines in the Pascal's Triangle. That equation isn't applicable to $n<0$; but here's a similar one valid in this case, concerning columns in the I.B.P.:

$$
\text { For } n<0: \quad F_{n}=2^{n+1} \sum_{i=0}^{\mathrm{int}\left(\frac{\mathrm{n}+1}{2}\right)} 5^{i}\left(\begin{array}{c}
2 i-2 \\
n-1
\end{array}\right) \text {. }
$$

There is, however, as it is known, another relationship (proved by finite induction) for $n \geq 0$ :

$$
F_{(n+1)}=\sum_{i=0}^{n}\left(\begin{array}{c}
n-i \\
i
\end{array}\right)
$$


concerning elements of ascending diagonals. Considering that, for $i>\frac{n}{2}$, all binomial coefficients result null, we may write, instead,

$$
\text { For } n \geq 0: \quad F_{(n+1)}=\sum_{i=0}^{\operatorname{int}\left(\frac{n}{2}\right)}\left(\begin{array}{c}
n-i \\
i
\end{array}\right) .
$$

Now, there's a similar equation for the left wing of Fibonacci sequence:

$$
\text { For } n<0: \quad F_{(n+1)}=\sum_{i=0}^{\operatorname{int}\left(\frac{\mathrm{n}}{2}\right)}\left(\begin{array}{c}
i \\
n-i
\end{array}\right) \text {. }
$$

For instance:

$$
\begin{aligned}
& n=-7: \quad F_{(-6)}=\sum_{i=0}^{-3}\left(\begin{array}{c}
i \\
n-i
\end{array}\right)=\left(\begin{array}{c}
0 \\
-7
\end{array}\right)+\left(\begin{array}{c}
-1 \\
-6
\end{array}\right)+\left(\begin{array}{c}
-2 \\
-5
\end{array}\right)+\left(\begin{array}{c}
-3 \\
-4
\end{array}\right)=0-1-4-3=-8 \\
& \text { and } \\
& n=-8: \quad F_{(-7)}=\sum_{i=0}^{-4}\left(\begin{array}{c}
i \\
n-i
\end{array}\right)=\left(\begin{array}{c}
0 \\
-8
\end{array}\right)+\left(\begin{array}{l}
-1 \\
-7
\end{array}\right)+\left(\begin{array}{l}
-2 \\
-6
\end{array}\right)+\left(\begin{array}{l}
-3 \\
-5
\end{array}\right)+\left(\begin{array}{c}
-4 \\
-4
\end{array}\right)=0+1+5+6+1=13 .
\end{aligned}
$$

b) For $n \leq 2$ and $0 \leq k \leq-n$, the sum of the elements of a descending diagonal is null:

$$
\sum_{k=0}^{n}\left(\begin{array}{c}
n+k \\
k
\end{array}\right)=0
$$

Take, for instance, $n=-5$ :

$$
\begin{aligned}
\sum_{k=0}^{-5}\left(\begin{array}{c}
n+k \\
k
\end{array}\right) & =\left(\begin{array}{c}
-5 \\
0
\end{array}\right)+\left(\begin{array}{c}
-4 \\
1
\end{array}\right)+\left(\begin{array}{c}
-3 \\
2
\end{array}\right)+\left(\begin{array}{c}
-2 \\
3
\end{array}\right)+\left(\begin{array}{c}
-1 \\
-4
\end{array}\right)+\left(\begin{array}{l}
0 \\
5
\end{array}\right) \\
& =1-4+6-4+1+0=0 .
\end{aligned}
$$

\section{Conclusion}

After creating the concept of factorial $n$ of order $k$ and generalizing it to real numbers $n$ and integer $k$, we saw some applications of it, mainly the generalization of $\left(\begin{array}{l}n \\ k\end{array}\right)$ and the concept of semi-integer derivative for $y=x^{n}$. Finally, we used the generalized concept of $\left(\begin{array}{l}n \\ k\end{array}\right)$ to build the Integer Binomial Plan, an expansion ot Pascal's Triangle, and studied some of its remarkable properties.

\section{References}

Andrew Sage \& James Melsa. (1983). Chapter 2. In 'Introdução às Probabilidades e Processos Estocásticos' (pp.42-46). Portuguese edition: Editora Portuguesa de Livros Técnicos e Científicos, Lda, Lisboa (translation of the original Edition, 'An Introduction to Probability and Stochastic Processes' (C)1973 Prentice-Hall, Inc).

N. Piskounov. (1975). Series. In 'Cálculo Diferencial e Integral', Vol. 2 (pp.309-311). Portuguese 2nd edition: Lopes da Silva Editora, Porto (translation of the 4th Russian Edition).

Wolfram MATHWORLD 1, Factorial [Online] Available: http://mathworld.Wolfram.com/Factorial.html

Wolfram MATHWORLD 2, Double factorial [Online] Available: http://mathworld.Wolfram.com/DoubleFactorial.html

Wolfram MATHWORLD 3, Fibonacci Numbers http://mathworld.wolfram.com/FibonacciNumber.html.

Note 1. These generalizations must be done quite as well for complex numbers $n$.

Note 2. This conclusion is consistent with the fact that the generalization of $n$ ! to complex numbers,

$$
z !=\Gamma(z+1)=\int_{0}^{\infty} e^{-t} t^{z} d t
$$

where $\Gamma(z+1)$ is the gamma function, "defines $z$ ! for all complex values of $z$, except when $z$ is a negative integer, in which case is equal to complex infinity” (Wolfram 1). 
Note 3. The displayed equation for $(-8)_{(3)}$ ! - or, generally speaking, to apply equation (1) to negative integer $n-$ may seem controversial because it establishes the quotient of two infinite products, at first sight an indetermination. Is it then legitimate to cancel $(-11)$ ! in both the numerator and the denominator of $(-8)_{(3)}$ ! ? I think so. We may analyse the question as following. Consider the infinite sets $F(-8)$ and $F(-11)$ consisting of the elements of $(-8)$ ! and ( -11$)$ ! respectively. We may establish a Cantor's one-to-one correspondence - an identity correspondence - between all the elements in one set and the other except for $-8,-9$ and -10 in $(-8)$ !. In conclusion, all the common elements cancel each other in the quotient, except for these 3 numbers and that's why it must be $(-8)_{(3)} !=(-8) \cdot(-9) \cdot(-10)$. This resolves the indetermination. Remark that, in the same way, the equality $8_{(3)} !=8 ! / 5$ ! establishes a Cantor's one-to-one correspondence between the sets (finite, now) $F(8)$ and $F(5)$; the numbers 8,7 and 6 are the only ones that aren't common to both sets.

Note 4. For $n=0$, this expression is not valid because $0_{(1)} !=\frac{1}{(-1) !}$ and we cannot simply replace $0_{(1)}$ ! by 0 . Certainly, however, the equation (7) remains valid, since

$$
0_{(k)} !=0_{(1)} !(-1)_{(k-1)} !=\frac{0 !}{(-1) !} \frac{(-1) !}{(-k) !}=\frac{1}{(-k) !},
$$

which is correct. A similar remark may be done to the 2 nd particular case, considering that $0_{(0)} !=0 !=1$.

Note 5. It may seems worthless to establish the exponent of $(-1)^{a}$ if $n_{(k)}$ ! is null or infinite. But, having in mind the former remarks on this subject, it's easy to understand that, applying the formula (8) in certain calculus, despising the correct exponent, we may be conduced to false results. In particular, this theorem directly implies the important Property 5 on binomial coefficients studied in section 5 . For instance, $(-2)_{(-5)} !=(-1)^{6} \cdot(-4)_{(-5)} !$, both being infinite factorials, implies that

$$
\left(\begin{array}{l}
-2 \\
-5
\end{array}\right)=(-1)^{6} \cdot\left(\begin{array}{c}
-4 \\
-5
\end{array}\right)=-4
$$

Note 6. Remark that a proof by the method of finite induction, which would be much shorter and would seem quite natural, would easily conduce to the wrong conclusion that $a=k$ holds in all cases. The reasoning fails because it implicitly admits an equality which isn't always true.

Note 7. As we have seen before, this means that both the numbers, (-4)! and (-6)!, although infinite, are not equals. There is, besides, a very precise relationship between them.

Note 8: Remark that, for $(n+1) \geq 0$ and $k<0$, it results $(n+1)_{(k)} ! \neq 0$ and $\frac{1}{k !}=0$, which means that (and this is quite visible in the I.B.P.)

$$
\left(\begin{array}{c}
n+1 \\
k
\end{array}\right)=0 \Rightarrow \sum_{k=-\infty}^{+\infty}\left(\begin{array}{c}
n+1 \\
k
\end{array}\right)=\sum_{k=0}^{+\infty}\left(\begin{array}{c}
n+1 \\
k
\end{array}\right)=\sum_{k=0}^{n+1}\left(\begin{array}{c}
n+1 \\
k
\end{array}\right)
$$

because, on the other hand, all binomial coefficients with $k>(n+1)$ are also null.

Note 9. It has been precisely the generalization (17) of this equality that, in 1976 , led me to the concept of $n_{(k)}$ ! and its notation and, subsequently, to the generalization of binomial coefficient.

Note 10. This fact is coherent with the one, already pointed out, that the transitive property of multiplication isn't always valid for null or infinite binomial coefficients. 